\title{
ON INVERSE SCATTERING FOR THE KLEIN-GORDON EQUATION
}

\author{
BY \\ TOMAS P. SCHONBEK
}

\begin{abstract}
A scattering operator $S=S(V)$ is set up for the Klein-Gordon equation $\square u=m^{2} u(m>0)$ perturbed by a linear potential $V=V(x)$ to $\square u=m^{2} u+V u$. It is found that for each $R>0$ there exists a constant $c(R)$ (of order $R^{2-n}$ as $R \rightarrow+\infty$, $n=$ space dimension) such that if the $L_{1}$ and the $L_{q}$ norm of $V$ and $V^{\prime}$ are bounded by $c(R), V^{\prime}-V$ is either nonnegative or nonpositive, and $V^{\prime}-V$ is of compact support having diameter $\leqq R$, then $S\left(V^{\prime}\right) \neq S(V)$ or $V^{\prime}=V$. Here $q>n / 2$, and $c(R)$ may also depend on $q$.
\end{abstract}

1. The purpose of this paper is to find conditions under which the scattering operator for the Klein-Gordon equation

$$
\square u=m^{2} u, \quad \square=\Delta-\partial^{2} / \partial t^{2},
$$

$\Delta$ the $n$-dimensional Laplacian, perturbed by a linear potential $V=V(x)$ to

$$
\square u=m^{2} u+V u,
$$

determines the scatterer $V$. For reasons of simplicity, we assume $m>0$. Then we can assume $m=1$, which in fact we do.

We look at the equations above in a suitable Hilbert space setup and consider the scattering operator as a mapping $V \rightarrow S(V)$ from a Banach space of functions to a Banach space of operators.

In the next section, we begin by developing a certain amount of theory essential to our results. This theory is basically a restatement of the results of [5, Chapter X, $\S 5]$, in a slightly more general setup, which is convenient to our needs. In $\$ \S 3$ and 4 we introduce the Hilbert spaces $\boldsymbol{H}_{a}\left(a \in R^{1}\right)$ in which we will work, and construct the wave and scattering operators as operators in $\boldsymbol{H}_{0}$. In $\$ \$ 5$ and 6 we combine all three previous sections to obtain some estimates on the wave operators, which lead us finally to our main result, stated in Theorem 7.5. The notation which is not explicitly defined when first used is either standard or self-explanatory. If $u$ is a function on $R^{n}$, we always denote by $\hat{u}$ the Fourier transform of $u$ which comes from defining

$$
\hat{u}(\xi)=(2 \pi)^{-n / 2} \int_{R^{n}} \exp (-i\langle x, \xi\rangle) u(x) d x
$$

if $u$ is an $L_{1}$ function.

Received by the editors March 9, 1971 and, in revised form, July 14, 1971.

AMS 1970 subject classifications. Primary 35P25, 47A40, 81A48; Secondary 35J10, 35L05, 47A55. 
We believe that the method applied here is new, though essentially straightforward. It relies heavily on results from [5] and [7]. It might be called an "implicit function theory approach"; as such it merely asserts uniqueness of the potential within a certain "domain of potentials", without attempting to construct it. For a constructive approach to a closely related problem, we refer to [3] and the references therein. We also refer to [6] for a very complete treatment of the scattering and inverse scattering problem for the equation $\square u=0$ perturbed by an obstacle, and to [8].for a more physically oriented approach to scattering. Both [6] and [8] have an excellent list of references, to which we refer for more information about scattering and inverse scattering problems. Finally, we want to mention, and thank the referee for pointing this out, that [2] contains a much more general and complete treatment of most of the material of $\S 4$.

The paper constitutes a first generalization of the author's doctoral dissertation at the Massachusetts Institute of Technology, under the direction of Professor Irving E. Segal. The author finds it a pleasure to acknowledge his deep indebtness to Professor Segal, both as a teacher and as an advisor. The author also wishes to thank Drs. Franklin E. Schroeck and Kerris W. Thompson for many interesting conversations on the physical aspects of the problem.

2. In this section $\boldsymbol{H}$ will denote a separable Hilbert space, $H$ a selfadjoint operator in $\boldsymbol{H}$. Define the Friedrichs operators $\Gamma^{+}$and $\Gamma^{-}$, with domain and range in the space $\boldsymbol{B}(\boldsymbol{H})$ of all bounded linear operators on $\boldsymbol{H}$, as follows: $D\left(\Gamma^{ \pm}\right)$is the set of all bounded operators $T$ such that

$$
\lim (a \rightarrow \pm \infty) i \int_{0}^{a} \exp (i t H) T \exp (-i t H) d t
$$

exists in the strong operator topology. For such a $T$ define $\Gamma^{ \pm}(T)$ as that limit. We write

$$
\Gamma^{ \pm}(T)=i \int_{0}^{ \pm \infty} \exp (i t H) T \exp (-i t H) d t
$$

(cf. [5, Chapter X, §5.2]). We shall simply write $\Gamma$ instead of $\Gamma^{+}$or $\Gamma^{-}$if there is no possibility of confusion.

LEMma 2.1. Consider $\boldsymbol{B}(\boldsymbol{H})$ as a Banach space in the usual operator norm. Then $\Gamma$, as an operator in this Banach space, is closed.

Proof. From the characterization of the range and domain of $\Gamma$ given in $[5$, Chapter X, Lemma 5.3] it follows easily that the graph of $\Gamma$ is closed.

Write $\{E(\lambda)\}$ to denote the spectral family determined by $H$. From now on, we assume that $H$ is spectrally absolutely continuous, i.e. the nondecreasing function $(E(\lambda) u, u)$ is absolutely continuous for all $u \in H$. Here ( , ) denotes the inner product of $\boldsymbol{H}$. Then $(E(\lambda) u, v)$ will also be an absolutely continuous function for all 
$u$ and $v$ in $\boldsymbol{H}$. We write $\rho(u, v)(\lambda)$ to denote the a.e. defined integrable function obtained by differentiating $(E(\lambda) u, v)$ with respect to $\lambda$. We set

$$
\rho(u)(\lambda)=\rho(u, u)(\lambda), \quad\|u\|^{2}=\text { ess sup } \rho(u)(\lambda) .
$$

The next two lemmas state the basic properties of these objects.

LEMma 2.2. Let $u, v, w, y$ be elements of $\boldsymbol{H}$. Then

$$
\begin{gathered}
\rho(u, v)=\overline{\rho(v, u)}, \quad \rho(u) \geqq 0, \\
|\rho(u, v)(\lambda)|^{2} \leqq \rho(u)(\lambda) \cdot \rho(v)(\lambda),
\end{gathered}
$$

(c) $\int_{-\infty}^{+\infty}(\exp (i t H) u, v) \overline{(\exp (i t H) w, y)} d t=2 \pi \int_{-\infty}^{+\infty} \rho(u, v)(\lambda) \cdot \overline{\rho(w, y)(\lambda)} d \lambda$, if $\|u\|<\infty$ and $\|w\|<\infty$.

$$
\int_{-\infty}^{+\infty}|(\exp (i t H) u, v)|^{2} d t=2 \pi \int_{-\infty}^{+\infty}|\rho(u, v)(\lambda)|^{2} d \lambda \leqq 2 \pi\|u\| \cdot\|v\| \|
$$

$$
\left|(\rho(u)(\lambda))^{1 / 2}-(\rho(v)(\lambda))^{1 / 2}\right| \leqq(\rho(u-v)(\lambda))^{1 / 2} .
$$

Proof. (a) is immediate. For a proof of (b), see [5, Chapter X, Theorem 1.7]. The rest follow from these two, Parseval's Theorem and standard properties of the spectral decomposition of an operator.

LEMMA 2.3. Let $\left\{u_{n}\right\}$ be a sequence of elements of $\boldsymbol{H}$.

(i) If $u_{n} \rightarrow u$, then $\left(\rho\left(u_{n}\right)\right)^{1 / 2} \rightarrow(\rho(u))^{1 / 2}$ in the $L_{2}\left(R^{1}\right)$ norm.

(ii) If $\left\|u_{n}\right\|<\infty$, and $\left\{u_{n}\right\}$ is Cauchy in $\|\cdot\| \|$, then $\left(\rho\left(u_{n}\right)\right)^{1 / 2}$ is Cauchy in $L_{\infty}\left(R^{1}\right)$.

(iii) If $u_{n} \rightarrow u,\left\|u_{n}\right\|<\infty$ and $\left\{u_{n}\right\}$ is Cauchy in $\|\cdot\|$, then $\|u\|<\infty$ and $\left\|u_{n}-u\right\|$ $\rightarrow 0$.

Proof. (iii) is a consequence of (i) and (ii), but (i) and (ii) follow from inequality (e) of Lemma 2.2.

We now want to perturb $H$ by an operator $A$, of the following type:

Assume given a $\sigma$-finite measure space $(M, M, \mu)$ and two measurable mappings $x \rightarrow f_{x}, x \rightarrow g_{x}$ of $M$ into $\boldsymbol{H}$ such that

$$
\begin{aligned}
\int_{M}\left\|f_{x}\right\|^{2} d \mu(x)<\infty, & \int_{M}\left\|g_{x}\right\|^{2} d \mu(x)<\infty, \\
\int_{M}\left\|f_{x}\right\|^{2} d \mu(x)<\infty, & \int_{M}\left\|g_{x}\right\|^{2} d \mu(x)<\infty, \\
x \rightarrow \rho\left(f_{x}\right), & x \rightarrow \rho\left(g_{x}\right)
\end{aligned}
$$

are measurable mappings from $M$ into $L_{\infty}\left(R^{1}\right)$.

We define the operator $A$ by

$$
A=\int_{M}\left(, g_{x}\right) f_{x} d \mu(x)
$$


i.e. for $u \in \boldsymbol{H}$ we set $A u=\int_{M}\left(u, g_{x}\right) f_{x} d \mu(x)$. Since the mapping

$$
x \rightarrow T_{x}, \quad T_{x} u=\left(u, g_{x}\right) f_{x},
$$

is an integrable $\boldsymbol{B}(\boldsymbol{H})$-valued function by (2.1), we have $A^{*}=\int_{M}\left(, f_{x}\right) g_{x} d \mu(x)$.

Assume furthermore

$$
\begin{aligned}
& \alpha^{2}=\operatorname{ess} \sup \int_{M} \rho\left(f_{x}\right) d \mu(x)<\infty, \\
& \beta^{2}=\operatorname{ess} \sup \int_{M} \rho\left(g_{x}\right) d \mu(x)<\infty .
\end{aligned}
$$

For $u, v$ in $H$, set $\sigma^{ \pm}(u, v)(\lambda)$ equal to the $L_{2}$-lim for $a \rightarrow \pm \infty$ of

$$
(2 \pi)^{1 / 2} \int_{0}^{a} \exp (i t \lambda)(\rho(u, v))^{\wedge}(t) d t
$$

where

$$
(\rho(u, v))^{\wedge}(t)=(2 \pi)^{-1 / 2} \int_{-\infty}^{+\infty} \exp (-i t \lambda) \rho(u, v)(\lambda) d \lambda
$$

Then, setting

$$
\left(G_{\delta}^{ \pm} \rho\right)(\lambda)=(2 \pi i)^{-1} \int_{-\infty}^{+\infty} \rho(\mu)(\mu-\lambda \mp i \delta)^{-1} d \mu,
$$

we also have $\sigma^{ \pm}(u, v)=G^{ \pm} \rho(u, v)$, with $G^{ \pm}=\mathrm{s}-\lim (\delta \downarrow 0) G_{\delta}^{ \pm}$.

We let $\sigma(u, v)$ stand for either $\sigma^{+}(u, v)$ or $\sigma^{-}(u, v)$ if there is no need to distinguish between them, or if there is otherwise no risk of confusion.

We define an operator in $L_{2}(M)$ as follows:

$$
[T(\lambda) h](x)=\int_{M}\left|\sigma\left(g_{x}, f_{y}\right)(\lambda)\right| h(y) d \mu(y) .
$$

Assume also given an orthogonal projection $\boldsymbol{P}$ in $\boldsymbol{H}$ such that $\boldsymbol{P}$ commutes with $H$, and such that if $b_{n}$ is the maximum of

$$
\text { ess } \sup _{\lambda}\|T(\lambda)\|^{n} \int_{M} \rho\left(P g_{x}\right) d \mu(x)
$$

and

$$
\operatorname{ess} \sup _{\lambda}\|T(\lambda)\|^{n} \int_{M} \rho\left(P f_{x}\right) d \mu(x)
$$

then

$$
\delta_{0}=\left[\lim \sup _{n}\left(b_{n}\right)^{1 / n}\right]^{-1}>0
$$

The main results of this section may be summarized as follows: 
THEOREM 2.4. Under all hypotheses above,

1. The operator $A$ defined by (2.4) is in $D(\Gamma)$, and $\Gamma(A) u=\int_{M} \sigma\left(u, g_{x}\right)(H) f_{x} d \mu(x)$, for $u \in \boldsymbol{H}$.

2. $\|\Gamma(A)\| \leqq 2 \pi \alpha \beta$.

3. Defining inductively

$$
\begin{array}{ll}
\Omega_{0}=P, & \Omega_{n+1}=\Gamma\left(A \Omega_{n}\right), \\
Z_{0}=P, & Z_{n+1}=-\Gamma\left(Z_{n} A\right) ;
\end{array}
$$

the series

$$
\Omega(x)=\sum_{n=0}^{\infty} x^{n} \Omega_{n}, \quad Z(x)=\sum_{n=0}^{\infty} x^{n} Z_{n}
$$

converge uniformly in operator norm in the disc $|x| \leqq \delta_{0}$. Here the symbol $\Omega$ actually stands for two operators, $\Omega_{+}$and $\Omega_{-}$. Similarly, $Z$ represents the operators $Z_{+}$ and $Z_{-}$.

4. The analytic operator valued functions $\Omega(x)$ and $Z(x)$ are solutions of the equations

$$
\Omega=P+\mathrm{x} \Gamma(A \Omega), \quad Z=P-\mathrm{x} \Gamma(Z A),
$$

respectively. Furthermore, for each $\mathrm{x}$ in the disc, we have $Z(\mathrm{x}) \Omega(\mathrm{x})=P$, for any pair of solutions of these equations. If $P=I$, then we also have $\Omega(x) Z(x)=I$, and the solutions to these equations are unique.

5. If the group generated by $i(H+x A)$ is uniformly bounded, then the solution of the first equation in 4 is uniquely determined by

$$
\Omega(\mathrm{x})=\mathrm{s}-\lim (t \rightarrow \pm \infty) \exp (i t(H+\mathrm{x} A)) \cdot \exp (-i t H)
$$

Proof. 1. It is proved in [5, Chapter X, (5.26)] that $\Gamma\left(T_{x}\right) u=i \sigma\left(u, g_{x}\right)(H) f_{x}$, where $T_{x}$ is the operator defined in (2.5). The rest follows from Lemma 2.1.

2. Essentially the same proof as in [5, Chapter X, Lemma 5.11].

3. Assuming $\Omega_{k}$ defined for $k=0,1, \ldots, n$ such that $\Omega_{k} P=\Omega_{k}, A \Omega_{k-1} \in D(\Gamma)$, $\Gamma\left(A \Omega_{k-1}\right)=\Omega_{k}$, we want to show $A \Omega_{n}$ is in $D(\Gamma)$. Since

$$
A \Omega_{n}=\int_{M}\left(, g_{n, x}\right) f_{x} d \mu(x)
$$

with $g_{n, x}=\Omega_{n}{ }^{*} g_{x}$; by part 2 of this lemma it suffices to show that

$$
\beta_{n}^{2}=\operatorname{ess} \sup _{\lambda} \int_{M} \rho\left(g_{n, x}\right) d \mu(x)<\infty
$$

Now

$$
g_{n, x}=\Omega_{n}{ }^{*} g_{x}=\left[\Gamma\left(A \Omega_{n-1}\right)\right]^{*} g_{x}=-\Gamma\left[\left(A \Omega_{n-1}\right)^{*}\right] g_{x}
$$


Using part 1 of this lemma, it is quite easy to show that if $v=\Gamma(T) u$, with $T=\int_{M}\left(, g^{\prime}{ }_{x}\right) f^{\prime}{ }_{x} d \mu(x)$, an operator of the same type as $A$ above, then

$$
\rho(v)=\iint \sigma\left(u, g_{x}^{\prime}\right) \overline{\sigma\left(u, g_{y}^{\prime}\right)} \rho\left(f_{x}^{\prime}, f^{\prime}{ }_{y}\right) d \mu(x) d \mu(y) .
$$

An application of Lemma 2.2(b) gives

$$
\rho(v) \leqq\left[\int_{M}\left|\sigma\left(u, g_{x}^{\prime}\right)\right|\left(\rho\left(f_{x}^{\prime}\right)\right)^{1 / 2} d \mu(x)\right]^{2} .
$$

In our case letting $T$ be the operator $\left(A \Omega_{n-1}\right)^{*}$, we get

$$
\rho\left(g_{n, x}\right)(\lambda) \leqq\left[T(\lambda)\left(\rho\left(g_{n-1, y}\right)(\lambda)\right)^{1 / 2}\right]^{2}(x),
$$

with $T(\lambda)$ the operator in $L_{2}(M)$ defined above. Thus

$$
\beta_{n}^{2} \leqq \operatorname{ess} \sup _{\lambda}\|T(\lambda)\|^{2} \int \rho\left(g_{n-1, y}\right) d \mu(y)
$$

and since $g_{0, x}=P g_{x}$, it follows that

$$
\beta_{n}^{2} \leqq \operatorname{ess}^{2} \sup _{\lambda}\|T(\lambda)\|^{2 n} \int \rho\left(P g_{x}\right)(\lambda) d \mu(x)
$$

By parts 1 and 2 of this lemma, $A \Omega_{n} \in D(\Gamma)$ and defining $\Omega_{n+1}=\Gamma\left(A \Omega_{n}\right)$, we have $\left\|\Omega_{n+1}\right\| \leqq 2 \pi \alpha \beta_{n}$. The convergence of the series for $\Omega(x)$ now follows from the definition of $\delta_{0}$. Since everything is symmetric in $f_{x}$ and $g_{x}$, the convergence of the series for $Z(x)$ is proved similarly.

4. That $\Omega(x)$ and $Z(x)$ are solutions of the equations is again an application of Lemma 2.1 and the convergence in operator norm of their series. The uniqueness of these solutions in case $P=I$ follows as in [5, Chapter X, Lemma 5.14].

5. The proof of this follows the same lines as the proof of [5, Chapter $X$, Theorem 5.8]. Notice that all arguments go through if the group generated by the perturbed operator is simply bounded. We also get

$$
(H+\mathrm{x} A) \Omega(\mathrm{x})=\Omega(\mathrm{x}) H .
$$

3. In this section, we describe the Hilbert spaces and operators with which we want to work. The notation introduced in this section will be used throughout.

Denote by $B$ the selfadjoint, nonnegative operator $(I-\Delta)^{1 / 2}$ in $L_{2}\left(R^{n}\right)$, with domain

$$
D(B)=\left\{u: \int\left(1+|\xi|^{2}\right)|\hat{u}(\xi)|^{2} d \xi<\infty\right\}
$$

We assume that the space dimension $n$ is odd, $n \geqq 3$.

In $D\left(B^{a}\right)$ (the domain of $B^{a}, a \in R^{1}$ ), we introduce the inner product

$$
[u, v]_{a}=\left\langle B^{a} u, B^{a} v\right\rangle,
$$


where $\langle$,$\rangle denotes the usual L_{2}$ inner product. Denote by $\left[D\left(B^{a}\right)\right]$ the Hilbert space completion of $D\left(B^{a}\right)$ in this inner product and notice that $D\left(B^{a}\right)$ is already complete if $a \geqq 0$.

Write

$$
\boldsymbol{H}_{a}=\left[D\left(B^{a+1}\right)\right] \oplus\left[D\left(B^{a}\right)\right]
$$

to denote the Hilbert space of ordered pairs $\{f, g\}\left(f \in\left[D\left(B^{a+1}\right)\right], g \in\left[D\left(B^{a}\right)\right]\right)$, inner product $(,)_{a}$ given by

$$
\left(\left\{u, u^{\prime}\right\},\left\{v, v^{\prime}\right\}\right)_{a}=[u, v]_{a+1}+\left[u^{\prime}, v^{\prime}\right]_{a} .
$$

In all notations, we drop the subindex " $a$ " in case $a=0$. For instance, we write $\boldsymbol{H}$ for $\boldsymbol{H}_{0}$, etc. We use, without always explicitly stating them, certain properties of these spaces which are immediate consequences of corresponding standard properties of the Sobolev spaces $\left[D\left(B^{a}\right)\right]$.

In $\boldsymbol{H}_{a}$ we define the selfadjoint operator $H$ by

$$
D(H)=H_{a+1}, \quad i H=\left[\begin{array}{cc}
0 & I \\
-B^{2} & 0
\end{array}\right] .
$$

This allows us to write the Klein-Gordon equation in $\boldsymbol{H}_{a}$ in the form $d u / d t=i H u$.

Let $V=V(x)$ be a real-valued function on $R^{n}$ such that the operator $A(V)$ in $H$, defined by

$$
i A(V)=\left[\begin{array}{cc}
0 & 0 \\
-M_{V} & 0
\end{array}\right]
$$

is $H$-bounded (cf. [3, Chapter IV, §1]). $M_{V}$ denotes the operator of multiplication by $V$. By the first inequality in the theorem of Sobolev and Kondrashov, as stated in [1, Part II, 5.3], it can easily be seen that $V \in L_{p}\left(R^{n}\right)$ suffices for $H$-boundedness, $p=\max (2, n / 2)$.

With this operator, our perturbed equation becomes, in $\boldsymbol{H}, d u / d t=i[H+A(V)] u$.

Another operator we want to consider in $\boldsymbol{H}$ is the operator $A_{\varepsilon}(V), \varepsilon>0, V$ as before. Define

$$
i A_{\varepsilon}=i A_{\varepsilon}(V)=\left[\begin{array}{cc}
0 & 0 \\
-\gamma_{\varepsilon} M_{V} \gamma_{\varepsilon} & 0
\end{array}\right]
$$

with $\gamma_{\varepsilon}$ defined by

$$
\left[\gamma_{\varepsilon} u\right]^{\wedge}(\xi)=\left(1+\varepsilon|\xi|^{2}\right)^{-n / 2} \hat{u}(\xi) .
$$

This operator has the following properties:

LEMMA 3.1. a. $\gamma_{\varepsilon}$ is a bounded, selfadjoint nonnegative operator on $L_{2}\left(R^{n}\right)$, of norm $\leqq 1$.

b. As an operator on $L_{2}\left(R^{n}\right)$, s-lim $(\varepsilon \downarrow 0) \gamma_{\varepsilon}=I$.

c. $\gamma_{\varepsilon}$ commutes with any Baire function of $B$.

d. If $g \in L_{1}\left(R^{n}\right)$, then so does $\gamma_{\varepsilon} g$, and the $L_{1}$-norm of $\gamma_{\varepsilon} g$ is bounded by $k$ times the $L_{1}$-norm of $g$, with $k$ a constant independent of $\varepsilon$. 
Proof. a. Immediate from Parseval.

b. Let $f \in L$. Denoting here by \|\| the $L_{2}$-norm,

$$
\left\|\gamma_{\varepsilon} f-f\right\|^{2}=\left\|\left(\gamma_{\varepsilon} f\right)^{\wedge}-\hat{f}\right\|^{2}=\int_{R^{n}}\left[\left(1+\varepsilon|\xi|^{2}\right)^{-n / 2}-1\right]|f(\xi)|^{2} d \xi
$$

and the last expression goes to 0 as $\varepsilon \downarrow 0$, by Lebesgue's dominated convergence theorem.

c. Immediate, since $\gamma_{\varepsilon}$ is itself a Baire function of $B$.

d. There exists $f \in L_{1}\left(R^{n}\right)$ such that $\hat{f}(\xi)=\left(1+|\xi|^{2}\right)^{-n / 2}$. In fact, by Parseval, there certainly exists such an $f \in L_{2}\left(R^{n}\right)$. It is not difficult to see that

$$
f(x)=(2 \pi)^{-n / 2}|x|^{(2-n) / 2} \int_{0}^{\infty} r^{n / 2}\left(1+r^{2}\right)^{-n / 2} J_{(n-2) / 2}(r|x|) d r
$$

with $J_{j}$ the $j$ th Bessel function of the first kind. Using the iteration formulas for these Bessel functions and the easily proven fact that, for $j=($ integer $)+\left(\frac{1}{2}\right)$, $r^{j}\left(1+r^{2}\right)^{1-j} J_{j}(r|x|) \rightarrow 0$ as $r \rightarrow 0,+\infty$, for any fixed $x$, integration by parts of the last integral gives

$$
f(x)=(2 / \pi)^{1 / 2} c_{n}|x|^{-1} \int_{0}^{\infty}\left(1+r^{2}\right)^{-3 / 2} r \sin (r|x|) d r
$$

with $c_{n}^{-1}=\left[(2 \pi)^{n / 2}(n-2)(n-4) \cdots 5 \cdot 3\right]$. The last integral may be evaluated in terms of modified Bessel functions of the second kind. We obtain

$$
f(x)=(2 / \pi)^{1 / 2} c_{n}|x|^{-1} K_{0}(|x|) .
$$

It follows now from Schlömilch's integral formula (cf. [4, (22), p. 82]) that $f$ is $O[\exp (-|x|)]$, for $|x| \rightarrow \infty$. Since $f$, being an $L_{2}$ function, is locally in $L_{1}$, this proves that $f$ is in $L_{1}\left(R^{n}\right)$.

Set $f_{\varepsilon}(x)=\varepsilon^{-n / 2} f\left(x / \varepsilon^{1 / 2}\right)$. Then $\hat{f}_{\varepsilon}(\xi)=\left(1+\varepsilon|\xi|^{2}\right)^{-n / 2}$ and the rest follows with $k=L_{1}$-norm of $f$.

For $h$ a function on $R^{n}$, denote by $|h|_{p}$ the $L_{p}$-norm of $h$; set $A_{0}(V)=A(V)$.

LemmA 3.2. a. s-lim $(\varepsilon \downarrow 0) A_{\varepsilon}=A$, s-lim $(\varepsilon \downarrow 0) A_{\varepsilon}^{*}=A^{*}$

b. $H+A_{\varepsilon}$ and $H+A_{\varepsilon}{ }^{*}$ converge strongly in the generalized sense (cf. [5, Chapter VIII, 1.1]) to $H+A$ and $H+A^{*}$ respectively.

c. Assume $|V|_{n / 2}<\infty$. If either $V \geqq 0$, or $|V|_{n / 2}<1$, then $i\left(H+A_{\varepsilon}(V)\right)$ generates a group bounded uniformly in $\varepsilon, t\left(0 \leqq \varepsilon, t \in R^{1}\right)$.

d. $\left[\exp \left(i t\left(H+A_{\varepsilon}\right)\right)\right]^{*}=\exp \left(-i t\left(H+A_{\varepsilon}^{*}\right)\right)(\varepsilon \geqq 0)$.

e.

$$
\begin{aligned}
\mathrm{s}-\lim (\varepsilon \downarrow 0) \exp \left(i t\left(H+A_{\varepsilon}\right)\right) & =\exp (i t(H+A)), \\
\mathrm{s}-\lim (\varepsilon \downarrow 0) \exp \left(i t\left(H+A_{\varepsilon}{ }^{*}\right)\right) & =\exp \left(i t\left(H+A^{*}\right)\right),
\end{aligned}
$$

the convergence being uniform in $t$, for $t$ ranging over compact intervals. 
Proof. $a$ is an easy consequence of Lemma 3.1. Notice that

$$
A_{\varepsilon}^{*}\{f, g\}=\left\{-B^{-2} \gamma_{\varepsilon} M_{V} \gamma_{\varepsilon} g, 0\right\}
$$

(setting also $\gamma_{0}=I$ ).

b is a straightforward application of [5, Chapter VIII, Theorem 1.5].

c. Let $u \in \boldsymbol{H}$, set

$$
\begin{aligned}
& u(t)=\left\{u_{1}(t), u_{2}(t)\right\}=\exp \left(i t\left(H+A_{\varepsilon}\right)\right) u, \\
& E(t)=\|u(t)\|^{2}+\left\langle\gamma_{\varepsilon} M_{v} \gamma_{\varepsilon} u_{1}(t), u_{1}(t)\right\rangle .
\end{aligned}
$$

Assuming $u \in D\left(H+A_{\varepsilon}\right)=D(H)$, it makes sense to differentiate the last expression with respect to $t$; we obtain $E^{\prime}(t)=0$, hence $E(t)=E(0)$, for all $t$.

From Sobolev's inequality (cf. [1, Part II, Chapter 5, §5.3]) it follows, for $p=2 n /(n-2), h \in L_{p}$ :

$$
|h|_{p} \leqq \text { const }\left.\cdot B^{2} h\right|_{2} \text {. }
$$

Thus

$$
\begin{aligned}
\left\langle\gamma_{\varepsilon} M_{V} \gamma_{\varepsilon} u_{1}(t), u_{1}(t)\right\rangle & =\left\langle M_{V} \gamma_{\varepsilon} u_{1}(t), \gamma_{\varepsilon} u_{1}(t)\right\rangle \leqq|V|_{n / 2}\left|\gamma_{\varepsilon} u_{1}(t)\right|_{p}^{2} \\
& \leqq|V|_{n / 2}\left|B^{2} \gamma_{\varepsilon} u_{1}(t)\right|_{2}^{2} \leqq|V|_{n / 2}\|u(t)\|^{2}
\end{aligned}
$$

This implies

$$
\left(1-|V|_{n / 2}\right)\|u(t)\|^{2} \leqq E(t) \leqq\left(1+|V|_{n / 2}\right)\|u(t)\|^{2}
$$

Thus

$$
\begin{aligned}
\|u(t)\|^{2} & \leqq E(t)=E(0) \leqq\left(1+|V|_{n / 2}\right)\|u\|^{2}, \quad \text { if } V \geqq 0 ; \\
\|u(t)\|^{2} & \leqq\left(1-|V|_{n / 2}\right)^{-1} E(t)=\left(1-|V|_{n / 2}\right)^{-1} E(0) \\
& \leqq\left(1-|V|_{n / 2}\right)^{-1}\left(1+|V|_{n / 2}\right)\|u\|^{2}, \quad \text { if }|V|_{n / 2}<1 .
\end{aligned}
$$

Since $D(H)$ is dense in $\boldsymbol{H}$, these inequalities must hold for all $\boldsymbol{u} \in \boldsymbol{H}$.

d. This is immediate.

e follows from $b$ and [5, Chapter IX, Theorem 2.16].

4. In this section, we assume $V \in L_{n / 2}$ and $V \geqq 0$, or $|V|_{n / 2}<1$.

LEMMA 4.1. Let $a>(n+2) / 4$. Set

$$
Q_{a}=\left\{u \in H: u=\left\{u_{1}, u_{2}\right\} ; B^{2 a} u_{1}, B^{2 a-1} u_{2} \in L_{1}\right\} .
$$

(i) $Q_{a}$ is dense in $H$.

(ii) If $u \in Q_{a}$, then $\left\|A_{\varepsilon} \exp (-i s H) u\right\| \leqq f_{u}(s)$, with $f_{u}$ some integrable function on the real line, which does not depend on $\varepsilon$.

Proof. (i) Immediate.

(ii) Let $W_{t, a}$ be the function defined in the first paragraph of [7], so that if $u \in Q_{a}$ and we set $u(t)=\exp (-i t H) u=\left\{u_{1}(t), u_{2}(t)\right\}$, we have

$$
u_{1}(t)=\left(\frac{1}{2}\right)\left[W_{-t, a}^{*} v_{1}+W_{t, a}^{*} v_{2}\right]
$$

with $v_{1}=B^{2 a} u_{1}+i B^{2 a-1} u_{2}, v_{2}=B^{2 a} u_{1}-i B^{2 a-1} u_{2}$. 
From the definition of $W_{t, a}$ as the function whose Fourier transform at the point $y$ is $\left(1+|y|^{2}\right)^{-a} \exp \left(-i t\left(1+|y|^{2}\right)^{1 / 2}\right)$, it is clear that $W_{t, a} \in L_{\infty}\left(R^{n}\right)$. By [7, Corollary 5.2], $\left|W_{t, a}\right|_{\infty}=O\left(|t|^{-n / 2}\right)$, for $|t| \rightarrow \infty$. Thus

$$
\left|W_{t, a}\right|_{\infty} \leqq \text { const }(1+|t|)^{-n / 2}, \quad t \in R^{1} .
$$

Applying the Hölder-Young inequalities to the expression for $u_{1}(t)$, we get

$$
\left|u_{1}(t)\right|_{\infty} \leqq \text { const }(1+|t|)^{-n / 2}\left(\left|B^{2 a} u_{1}\right|_{1}+\left|B^{2 a-1} u_{1}\right|_{1}\right) .
$$

We use this in the following estimate, the constant $k$ is the one of Lemma 3.1; the steps are justified by parts $\mathrm{c}$ and $\mathrm{d}$ of Lemma 3.1 :

$$
\begin{aligned}
\|A \exp (-i s H) u\| & =\left|\gamma_{\varepsilon} M_{V} \gamma_{\varepsilon} u_{1}(s)\right|_{2} \\
& \leqq\left|V \gamma_{\varepsilon}[\exp (-i s H) u]_{1}\right|_{2}=\left|V\left[\exp (-i s H)\left\{\gamma_{\varepsilon} u_{1}, \gamma_{\varepsilon} u_{2}\right\}\right]_{1}\right|_{2} \\
& \leqq|V|_{2}\left|\left[\exp (-i s H)\left\{\gamma_{\varepsilon} u_{1}, \gamma_{\varepsilon} u_{2}\right\}\right]_{1}\right|_{\infty} \\
& \leqq \text { const }|V|_{2}(1+|s|)^{-n / 2}\left(\left|B^{2 a} \gamma_{\varepsilon} u_{1}\right|_{1}+\left|B^{2 a-1} \gamma_{\varepsilon} u_{2}\right|_{1}\right) \\
& \leqq \text { const } k|V|_{2}(1+|s|)^{-n / 2}\left(\left|B^{2 a} u_{1}\right|_{1}+\left|B^{2 a-1} u_{2}\right|_{1}\right),
\end{aligned}
$$

and this proves the lemma.

Reasoning as in [5, Chapter $X$, Theorem 3.7], we get as a first consequence of this lemma,

LEMMA 4.2. The operators

$$
W^{\varepsilon}(V)=\mathrm{s}-\lim (t \rightarrow \pm \infty) \exp \left(i t\left(H+A_{\varepsilon}\right)\right) \exp (-i t H) \quad(\varepsilon \geqq 0)
$$

exist on $\boldsymbol{H}$.

If $\varepsilon=0$, we simply write $W_{ \pm}(V)$. Another consequence of Lemma 4.1 is the following

LEMMA 4.3. $\mathrm{s}-\lim (\varepsilon \downarrow 0) W^{\varepsilon}(V)=W_{ \pm}(V)$.

Proof. It is not difficult to see that

$$
W^{\varepsilon}+(V) u=u+i \int_{0}^{+\infty} \exp \left(i t\left(H+A_{\varepsilon}\right)\right) A_{\varepsilon} \exp (-i t H) u d t
$$

for $\varepsilon \geqq 0$. This integral has to be interpreted as the strong limit of the corresponding finite integrals.

Let $u \in Q_{a}$. Lemma 4.1 then implies that the integrand above is integrable in the Lebesgue sense and provides a uniform $L_{1}$-estimate of its norm. Thus we may apply Lebesgue's dominated convergence theorem to the convergence given in part e of Lemma 3.2 to obtain

$$
\lim (\varepsilon \downarrow 0) W^{\varepsilon}+(V) u=u+i \int_{0}^{\infty} \exp (i t(H+A)) A \exp (-i t H) u d t .
$$

This proves the "+" case of the lemma. The "_" case is entirely similar. 
The operators $W^{8}{ }_{ \pm}$defined in Lemma 4.2 are called the wave operators for the perturbation $A_{\varepsilon}(\varepsilon \geqq 0)$.

In the same way as we proved Lemma 4.2 , we can also prove (by an obvious modification of Lemma 4.1), that

$$
W^{\varepsilon+}=\mathrm{s}-\lim (t \rightarrow+\infty) \exp \left(i t\left(H+A_{\varepsilon}{ }^{*}\right)\right) \exp (-i t H)
$$

exists. We use this operator to define

$$
S_{\varepsilon}(V)=\left[W^{\varepsilon+}(V)\right]^{*} W^{\varepsilon}-(V) .
$$

We call $S_{\delta}(V)$ the scattering operator corresponding to the perturbation $A_{8}(V)$.

5. We now apply the results of $\$ 2$ to the situation described in $\$ \$ 3$ and 4 .

THEOREM 5.1. The operator $H$ is spectrally absolutely continuous in each $\boldsymbol{H}_{a}$, with spectral family $\{E(\lambda)\}$ given by

$$
E(\lambda)=\left[\begin{array}{cc}
P(\lambda) & -i B^{-1} Q(\lambda) \\
i B Q(\lambda) & P(\lambda)
\end{array}\right]
$$

where $P(\lambda), Q(\lambda)$ are defined by

$$
\begin{aligned}
{[P(\lambda) f]^{\wedge}(\xi) } & =\left(\frac{1}{2}\right) \chi_{\lambda} f(\xi), & & \lambda<-1, \\
& =\left(\frac{1}{2}\right) f(\xi), & & -1 \leqq \lambda<1, \\
& =f(\xi)-\left(\frac{1}{2}\right) \chi_{\lambda} f(\xi), & & 1 \leqq \lambda ; \\
{[Q(\lambda) f]^{\wedge}(\xi) } & =-\left(\frac{1}{2}\right) \chi_{\lambda} f(\xi), & & |\lambda|>1, \\
& =-\left(\frac{1}{2}\right) f(\xi), & & |\lambda| \leqq 1 ;
\end{aligned}
$$

with $\chi_{\lambda}=\chi_{\lambda}(\xi)$, the characteristic function of the set $\left\{\eta \in R^{n}:|\eta|>\left(\lambda-{ }^{2} 1\right)^{1 / 2}\right\}$.

Proof. It is straightforward to check that the family $\{E(\lambda)\}$ defined above is the spectral decomposition of some selfadjoint, spectrally absolutely continuous operator $H^{\prime}$. Then check that $(\exp (i t H) u, v)_{a}=\left(\exp \left(i t H^{\prime}\right) u, v\right)_{a}$ for all $u, v \in \boldsymbol{H}_{a}$, $t \in R^{1}$.

Given $u, v \in \boldsymbol{H}_{a}$ set

$$
\begin{aligned}
& \rho^{a}(u, v)(\lambda)=(d / d \lambda)(E(\lambda) u, v)_{a}, \quad \rho^{a}(u)(\lambda)=\rho^{a}(u, u)(\lambda), \\
& \|u\|_{a(M)}^{2}=\underset{|\lambda|<M}{\operatorname{ess} \sup ^{a} \rho^{a}(u)(\lambda),} \quad\|u\|_{a}=\|u\|_{a(\infty)},
\end{aligned}
$$

for $a \in R^{1}, 0<M \leqq \infty$. We follow our usual convention of dropping the subindex “ $a$ " in case $a=0$, for instance $\|u\|_{(M)}=\|u\|_{O(M)}$.

Since $|(u, v)| \leqq\|u\|_{a}\|v\|_{-a}$, we may define $(u, v)$ and consequently $\rho(u, v)$ for $u \in \boldsymbol{H}_{a}, v \in \boldsymbol{H}_{-a}, a$ arbitrary. The last written inequality may be used instead of the Cauchy inequality to prove

$$
|\rho(u, v)(\lambda)|^{2} \leqq \rho^{a}(u)(\lambda) \rho^{-a}(v)(\lambda)
$$

in the same way as inequality (b) of Lemma 2.2. 
From now on we assume throughout that $V$ is an element of $L_{1}\left(R^{n}\right) \cap L_{n / 2}\left(R^{n}\right)$; $V \geqq 0$ or $|V|_{n / 2}<1$. Define two functions $V_{1}, V_{2}$ on $R^{n}$ by

$$
V_{1}(x) V_{2}(x)=V(x), \quad V_{1}(x)^{2}=V_{2}(x)^{2}=V(x) .
$$

For each $x \in R^{n}$, let $\zeta^{8}{ }_{x}$ be the $L_{2}$ function whose Fourier transform is

$$
(2 \pi)^{-n / 2}\left(1+\varepsilon|\xi|^{2}\right)^{-n / 2} \exp [-i\langle x, \xi\rangle]
$$

Define

$$
\Phi_{x}^{\varepsilon}=\left\{0, \zeta_{x}^{\varepsilon}\right\}, \quad \Psi_{x}^{\varepsilon}=\left\{B^{-2} \zeta_{x}^{\varepsilon}, 0\right\}
$$

Set

$$
f^{8}{ }_{x}=f_{x}^{\varepsilon}(V)=V_{1}(x) \Phi_{x}^{8}, \quad g_{x}^{\delta}=g_{x}^{\delta}(V)=V_{2}(x) \Psi_{x}^{8} .
$$

In this section, we assume $\varepsilon>0$.

Let $\omega_{n}$ be the area of the unit sphere in $R^{n}$, set $k=(n-2) / 2$ and, for $|\lambda|>1$, define

$$
h_{\varepsilon}(\lambda)=\frac{\omega_{n}}{2} \cdot \frac{\left(\lambda^{2}-1\right)^{k}}{(2 \pi)^{n}\left[1+\varepsilon\left(\lambda^{2}-1\right)\right]^{n}}
$$

LEMMA 5.2. The functions $\rho^{a}\left(\Phi_{x}^{\varepsilon}\right), \rho^{a}\left(\Psi_{x}^{\varepsilon}\right), \rho\left(\Psi_{x}^{\varepsilon}, \Phi_{y}^{\varepsilon}\right)$ are all $=0$ if $|\lambda|<1$, while

$$
\begin{gathered}
\rho^{a}\left(\Phi_{x}^{\varepsilon}\right)=h_{\varepsilon}(\lambda)|\lambda|^{2 a+1}, \quad|\lambda|>1 ; \\
\rho^{a}\left(\Psi_{x}^{\varepsilon}\right)=h_{\varepsilon}(\lambda)|\lambda|^{2 a-1}, \quad|\lambda|>1 ; \\
\rho\left(\Psi_{x}^{\varepsilon}, \Phi_{y}^{\varepsilon}\right)=\rho(\lambda) J_{k}\left(\left(\lambda^{2}-1\right)^{1 / 2}|x-y|\right)|x-y|^{-k}, \quad|\lambda|>1 ;
\end{gathered}
$$

with

$$
\rho(\lambda)=(i / 2) \operatorname{sgn} \lambda \frac{\left(\lambda^{2}-1\right)^{k / 2}}{(2 \pi)^{n}\left[1+\varepsilon\left(\lambda^{2}-1\right)\right]^{n}}
$$

and $J_{k}$ the kth Bessel function of the first kind.

Proof. This follows from Theorem 5.1 by straightforward computations.

THEOREM 5.3. The mappings $x \rightarrow f_{x}, x \rightarrow g_{x}$, from $R^{n}$ into $\boldsymbol{H}$, are Borel measurable and satisfy properties (2.1)-(2.3) and (2.6). Furthermore

$$
\int_{R^{n}}\left(, g^{8}{ }_{x}\right) f_{x}^{\varepsilon} d x=A_{\varepsilon}(V) .
$$

Proof. Properties (2.1)-(2.3) and (2.6) are easy consequences of $V \in L_{1}$ and (5.2), (5.3). Notice that $\rho\left(f_{x}^{8}\right)=|V(x)| \rho\left(\Phi_{x}^{\varepsilon}\right), \rho\left(g_{x}^{\varepsilon}\right)=|V(x)| \rho\left(\Psi_{x}^{8}\right)$.

To prove the integral expression for $A_{\varepsilon}(V)$, check it on smooth elements of $\boldsymbol{H}$. Since such elements are dense in $\boldsymbol{H}$, the theorem follows.

We set $\sigma(x, y ; \lambda)=\sigma\left(\Psi_{x}^{8}, \Phi_{y}^{8}\right)(\lambda)$, with $\sigma(u, v)$ defined as in $\$ 2$ for $u, v \in \boldsymbol{H}$. 
LEMMA 5.4. If $0<\varepsilon \leqq \frac{1}{2}$, then

$$
|\sigma(x, y ; \lambda)| \leqq \Lambda\left[1+\left|\lambda^{2}-1\right|^{(n-3) / 2}\right] p(|x-y|),
$$

with $\Lambda$ a constant depending only on $n$;

$$
\begin{aligned}
p(t) & =t^{2-n} & & \text { if } 0<t<1, \\
& =t^{-(1 / 2)(n-1)} & & \text { if } t>1 .
\end{aligned}
$$

Proof. Set $k=(n-3) / 2$, so that $(n-2) / 2$ becomes $k+\left(\frac{1}{2}\right)$. We use the fact that

with

$$
J_{k+(1 / 2)}(z)=P_{k}\left(1 / z^{1 / 2}\right) \sin z+Q_{k}\left(1 / z^{1 / 2}\right) \cos z,
$$

$$
P_{k}(\lambda)=\sum_{j=0}^{m} a_{j} \lambda^{4 j+1}, \quad Q_{k}(\lambda)=\sum_{j=0}^{m-1} b_{j} \lambda^{4 j+3}
$$

if $k=2 m$; or

$$
P_{k}(\lambda)=\sum_{j=0}^{m} a_{j} \lambda^{4 j+3}, \quad Q_{k}(\lambda)=\sum_{j=0}^{m} b_{j} \lambda^{4 j+1}
$$

if $k=2 m+1[9,17.24]$.

From this it is easy to see that $G_{\delta}^{+}$of the function defined by (5.4) may be written as a sum of terms of the form

$$
\text { const }(|x-y|)^{-n+p+1} \int_{-\infty}^{+\infty} \frac{\nu^{p} \exp (i v|x-y|)}{\left(1+\varepsilon \nu^{2}\right)^{n}\left(\nu^{2}+1-\gamma^{2}\right)} d \nu
$$

for $p$ ranging from 1 to $(n-1) / 2, \gamma=\gamma(\lambda, \delta)=\lambda+i \delta$. The integral is equal to the sum of the residues of the integrand in the upper half plane. These are located at $\nu=i / \varepsilon^{1 / 2}$ (pole of order $n$ ) and at $\nu=r$, where $r^{2}=(\lambda+i \delta)^{2}-1, \operatorname{Im}(r)>0$ (pole of order 1).

Claim. The residue at $\nu=i / \varepsilon^{1 / 2}$ is bounded by const $|x-y|^{1-p}$, the constant independent of $\varepsilon,|x-y|$ or $\lambda$. For this, notice that the residue in question is (up to constant factors) a sum of terms of the form

$$
\varepsilon^{-n} D^{h}\left(\nu^{p}\right) \times D^{j}\left[\left(\nu+i / \varepsilon^{1 / 2}\right)^{-n}\right] \times D^{k}[\exp (i v|x-y|)] \times D^{s}(d(\nu)),
$$

evaluated at $\nu=i / \varepsilon^{1 / 2}$, with $h+j+k+s=n-1, D^{h}$ denoting differentiation with respect to $v h$-times, and $d(v)=\left(v^{2}+1-\gamma^{2}\right)^{-1}$. One proves easily, by induction,

$$
\begin{aligned}
D^{2 m} d(\nu) & =\sum_{k=0}^{m} c_{k} \nu^{2 k} d(\nu)^{m+k+1}, \\
D^{2 m+1} d(\nu) & =\sum_{k=0}^{m} c_{k} \nu^{2 k+1} d(\nu)^{r+k+2} .
\end{aligned}
$$

Since

$$
\left|d\left(i / \varepsilon^{1 / 2}\right)\right| \leqq\left|\operatorname{Re}\left[d\left(i / \varepsilon^{1 / 2}\right)^{-1}\right]\right|^{-1} \leqq 2 \varepsilon,
$$

if $0<\varepsilon \leqq 1 / 2, \delta$ sufficiently small (this follows by considering the cases $\lambda^{2}-\delta^{2} \geqq 1$, $\lambda^{2}-\delta^{2} \leqq 1$ separately and realizing that we may assume $\lambda^{2}-\delta^{2} \geqq 0$ ); we obtain 
$\left|D^{s} d\left(i / \varepsilon^{1 / 2}\right)\right| \leqq$ const $\left(\varepsilon^{1 / 2}\right)^{s+2}$. Using this in $\left(^{*}\right)$ evaluated at $\nu=i / \varepsilon^{1 / 2}$, we see that $\left(^{*}\right)$ is bounded by

$$
\text { const } \begin{aligned}
|x-y|{ }^{k}\left(\varepsilon^{1 / 2}\right)^{-p-k+1} \exp \left(-|x-y| / \varepsilon^{1 / 2}\right) & \\
= & \text { const }|x-y|^{1-p}\left[\frac{|x-y|}{\varepsilon^{1 / 2}} \exp \left(-\frac{|x-y|}{(p+k-1) \varepsilon^{1 / 2}}\right)\right]^{p+k-1}
\end{aligned}
$$

Using the inequality $b \exp (-\tau b) \leqq \tau^{-1} e^{-1}$, valid for all $b, \tau>0$, we can further estimate $\left({ }^{* *}\right)$ by const $|x-y|^{1-p}$. This proves the claim.

Now letting $r$ be such that $r^{2}=\gamma^{2}-1, \operatorname{Im}(r)>0$, the residue at $r$ is

$$
\left(\frac{1}{2}\right) r^{p-1}\left(1+\varepsilon r^{2}\right)^{-n} \exp (i r|x-y|) \text {. }
$$

Again using $\varepsilon \leqq \frac{1}{2}$ and that $\lim (\delta \rightarrow 0) r^{2}=\lambda^{2}-1$, we may assume $\left|1+\varepsilon r^{2}\right| \geqq \frac{1}{2}$. Thus as $\delta \rightarrow 0$ the residue becomes bounded by $\left|\lambda^{2}-1\right|^{(p-1) / 2}$. Putting all this information together, it follows that

$$
|\sigma(x, y ; \lambda)| \leqq \text { const }\left[|x-y|^{2-n}+|x-y|^{(1-n) / 2}\left(\left|\lambda^{2}-1\right|^{1 / 2}+|x-y|^{-1}\right)^{(n-3) / 2}\right]
$$

and from this the assertion of the lemma follows.

We let the projection $P$ of $\S 2$ be the identity operator in $H$ if $n=3, P=P_{M}$ for $n>3(0<M<\infty)$, with $P_{M}$ defined as multiplication of each component by the characteristic function of the set $\{|\xi|<M\}$, in the Fourier transform space:

$$
\begin{aligned}
& P_{M}\{f, g\}=\left\{f^{\prime}, g^{\prime}\right\}, \\
& \hat{f}^{\prime}(\xi)=\hat{f}(\xi) \text { if }|\xi|<M, \\
&=0 \text { if }|\xi| \geqq M \\
& \hat{g}^{\prime}(\xi)=\hat{g}(\xi) \text { if }|\xi|<M, \\
&=0 \text { if }|\xi| \geqq M .
\end{aligned}
$$

It is clear that $P$ commutes with $H$. Also

$$
\begin{aligned}
\rho\left(P_{M} u, v\right)(\lambda) & =\rho(u, v)(\lambda) & & \text { if } \lambda^{2}<M^{2}+1, \\
& =0 & & \text { if } \lambda^{2}>M^{2}+1(n>3) .
\end{aligned}
$$

Thus, the $\delta_{0}$ of $\S 2$ may be estimated by $\Lambda^{\prime}\left[1+M^{n-3}\right]\left(|V|_{1}+|V|_{n / 2}\right)$ with $\Lambda^{\prime}$ a constant depending only on $n$. Notice that this estimate does not depend on $M$ if $n=3$.

Proof of estimate. Define the operator $T(\lambda)$ as in $\$ 2$ by

$$
\begin{aligned}
{[T(\lambda) h](x) } & =\int_{R^{n}}\left|\sigma\left(\dot{g}_{x}^{\varepsilon}, f_{y}^{\varepsilon}\right)(\lambda)\right| h(y) d y \\
& =\int_{R^{n}}|\sigma(x, y ; \lambda)| V_{2}(x) V_{1}(y) h(y) d y .
\end{aligned}
$$

By Lemma 5.4,

$$
|T(\lambda) h|_{2} \leqq \Lambda\left[1+\left|\lambda^{2}-1\right|^{(n-3) / 2}\right]\left(\left|V_{2}\left(g_{1} * V_{1} h\right)\right|_{2}+\left|V_{2}\left(g_{2} * V_{1} h\right)\right|_{2}\right),
$$


with $g_{1}(x)=|x|^{2-n}$ if $|x|<1,=0$ otherwise; $g_{2}(x)=|x|^{(1-n) / 2}$ if $|x| \geqq 1,=0$ otherwise.

Applying Hölder, Sobolev [1, Part II, §5.3c] and Hölder,

$$
\left|V_{2}\left(g_{1} * V_{1} h\right)\right|_{2} \leqq \text { const }\left|V_{1}\right|_{n}\left|V_{2}\right|_{n}|h|_{2}=\text { const }|V|_{n / 2}|h|_{2} \text {. }
$$

Just by Hölder (since $\left|g_{2}(x)\right| \leqq 1$ )

$$
\left|V_{2}\left(g_{2} * V_{1} h\right)\right|_{2} \leqq\left|V_{2}\right|_{2}\left|V_{1}\right|_{2}|h|_{2}=|V|_{1}|h|_{2} .
$$

Considering that

$$
\int_{R^{n}} \rho\left(P_{M} g_{x}^{\varepsilon}\right) d x=\int_{R^{n}} \rho\left(P_{M} f_{x}^{\varepsilon}\right) d x=0
$$

for $\lambda^{2}>M^{2}+1$, the estimate follows.

From now on, we denote by $\Lambda$ a constant which depends only on $n$, and into which we absorb all such constants appearing in the estimates of the sequel. Set

$$
\begin{gathered}
c(M)=\Lambda\left[1+M^{n-3}\right], \\
\Omega^{\varepsilon}{ }_{ \pm}(V)=W^{\varepsilon}{ }_{ \pm}(V) P \quad\left(P=I \text { if } n=3, P=P_{M} \text { if } n>3\right) .
\end{gathered}
$$

We obtain directly from Theorem 2.4, the last estimate and Hölder inequalities.

THEOREM 5.5. Let $q \geqq n / 2$, assume $|V|_{1}+|V|_{q}<c(M)^{-1}$. The series $\sum_{n=0}^{\infty} \Omega^{8 \pm}{ }_{n}(V)$, with terms defined by

$$
\Omega^{\varepsilon \pm}{ }_{0}(V)=P, \quad \Omega^{\varepsilon \pm}{ }_{n+1}(V)=\Gamma\left[A_{\varepsilon}(V) \Omega^{\varepsilon \pm}{ }_{n}(V)\right],
$$

converges in operator norm to $\Omega^{\varepsilon}{ }_{ \pm}(V)$. Furthermore, $\Omega^{\varepsilon}{ }_{ \pm}=P+\Gamma^{ \pm}\left(A_{\varepsilon} \Omega^{\varepsilon}{ }_{ \pm}\right)$.

Proof. Theorem 2.4 proves convergence of the series to a solution of the last equation above. Since the group generated by $i\left(H+A_{\varepsilon}\right)$ is uniformly bounded (Lemma 3.2c), it also follows from Theorem 2.4 that

$$
\Omega^{\varepsilon}{ }_{ \pm}=\mathrm{s}-\lim (t \rightarrow \pm \infty) \exp \left(i t\left(H+A_{\varepsilon}\right)\right) \exp (-i t H) P=W^{\varepsilon}{ }_{ \pm} P .
$$

As a final result of this section we want the following two properties of the operator $S_{\varepsilon}(V)$ defined by (4.3):

Lemma 5.6. Assume $\varepsilon>0,|V|_{1}+|V|_{q}<c(M)^{-1}, q \geqq n / 2$. Then

$$
S_{\varepsilon}(V) P=P-i \int_{-\infty}^{+\infty} \exp (i t H) A_{\varepsilon} \Omega^{\varepsilon}-\exp (-i t H) d t
$$

(the integral a weak operator limit), and

$$
\text { w-lim }(\varepsilon \downarrow 0) S_{\varepsilon}(V)=S(V) .
$$

Proof. From the equation satisfied by $\Omega^{\varepsilon}$, it is easy to see that

$$
\exp (i t H) \Omega^{\varepsilon}-\exp (-i t H)=P-i \int_{-\infty}^{t} \exp (i s H) A_{\varepsilon} \Omega^{\varepsilon}-\exp (-i s H) d s
$$


Thus, setting $W^{\varepsilon}(t)=\exp \left(i t\left(H+A_{\varepsilon}^{*}\right)\right) \exp (-i t H)$, we have for $u, v \in \boldsymbol{H}$,

$$
\begin{aligned}
\left(S_{\varepsilon} P u, v\right) & =\left(W^{\varepsilon+*} W_{-} P u, v\right)=\left(\Omega^{\varepsilon}-u, W^{\varepsilon+} v\right) \\
& =\lim (t \rightarrow+\infty)\left(\Omega^{\varepsilon}-u, W^{\varepsilon}(t) v\right) \\
& =\lim (t \rightarrow+\infty)\left(\exp (i t H) \Omega^{\varepsilon}-\exp (-i t H) u, v\right) \\
& =(P u, v)-i \lim (t \rightarrow+\infty) \int_{-\infty}^{t}\left(\exp (i s H) A_{\varepsilon} \Omega^{\varepsilon}-\exp (-i s H) u, v\right) d s
\end{aligned}
$$

and since the $\lim (t \rightarrow+\infty)$ exists, this proves the expression for $S_{\varepsilon}(V)$ given above.

Since $i\left(H+A_{\varepsilon}\right)$ and $i\left(H+A_{\varepsilon}{ }^{*}\right)$ generate groups which are uniformly bounded also in $\varepsilon$, it follows that $S_{\varepsilon}(V)$ is uniformly bounded in $\varepsilon$. Thus, to prove w-lim $(\varepsilon \downarrow 0) S_{\varepsilon}(V)=S(V)$, it is enough to do so on a dense domain.

Let $u \in H, v \in Q_{a}, Q_{a}$ defined by (4.1). Set

Then

$$
g_{\varepsilon}(s)=\left(\Omega^{\varepsilon}-\exp (-i s H) u, A_{\varepsilon}^{*} \exp (-i s H) v\right) .
$$

$$
\begin{aligned}
\left|g_{\varepsilon}(s)\right| & \leqq F_{v}(s)\left\|\Omega^{\varepsilon}-\exp (-i s H) u\right\| \\
& \leqq F_{v}(s)\left\|\Omega^{\varepsilon}-\right\|\|u\| \leqq \text { const }\|u\| F_{v}(s),
\end{aligned}
$$

where $F_{v}$ is the analogue for $A_{\varepsilon}{ }^{*}$ of the function $f_{u}$ for $A_{\varepsilon}$ obtained in Lemma 4.1(ii).

Thus, by Lemma 3.2a, Lemma 4.3 , the uniform boundedness in $\varepsilon$ of the wave operators and Lebesgue's dominated convergence theorem

$$
\lim (\varepsilon \downarrow 0) \int_{-\infty}^{+\infty} g_{\varepsilon}(s) d s=\int_{-\infty}^{+\infty} g_{0}(s) d s .
$$

This proves $S_{\varepsilon}(V) P \rightarrow S(V) P$ in the weak operator topology, completing the proof of the lemma if $n=3$. For $n>3$, notice that $\left\{P_{M} Q_{a}: M>0\right\}$ is dense in $\boldsymbol{H}$.

6. Fix $q>n / 2$. Define

$$
|V|=|V|(q)=|V|_{1}+|V|_{q}
$$

From now on we assume $|V|<c(M)^{-1}$.

LEMMA 6.1. Set $N^{2}=M^{2}+1$.

$$
\begin{aligned}
& \left\|\mid \Omega^{\varepsilon}(V)^{*} \Psi^{\varepsilon}{ }_{x}\right\|_{(N)} \leqq c(M) /(1-c(M)|V|), \\
& \left\|\left[\Omega^{8}(V)-P\right]^{*} \Psi^{s}{ }_{x}\right\|_{(N)} \leqq c(M)|V| /(1-c(M)|V|) .
\end{aligned}
$$

Proof. Since the series for $\Omega^{8}(V)$ given by Theorem 5.5 converges in the operator norm topology, we may write $\Omega^{8}(V)^{*}=\sum_{m=0}^{\infty} \Omega_{m}^{8}{ }_{m}^{*}(V)$, convergence again in norm topology.

Set $r_{m, x}=\Omega^{8}{ }_{m}{ }^{*} \Psi^{8}{ }_{x}$ ( $V$ will remain fixed for the rest of this proof.) We now proceed similarly as in the proof of Theorem 2.4.

By Theorem 5.5

$$
r_{m+1, x}=\Omega_{m+1}^{8}{ }^{*} \Psi_{x}^{\varepsilon}=\Gamma\left(A_{8} \Omega_{m}^{8}\right)^{*} \Psi_{x}^{s}=-\Gamma\left[\left(A_{\varepsilon} \Omega_{m}^{8}\right)^{*}\right] \Psi^{s}{ }_{x} .
$$


Proceeding as in (2.8),

$$
\rho\left(r_{m+1, x}\right)(\lambda) \leqq\left(\int_{R^{n}}|V(y)||\sigma(x, y ; \lambda)|\left(\rho\left(r_{m, y}\right)(\lambda)\right)^{1 / 2} d y\right)^{2} .
$$

Setting

$$
I_{m}(x ; \lambda)=\int_{R^{n}}|V(y)| p(|x-y|)\left(\rho\left(r_{m, y}\right)(\lambda)\right)^{1 / 2} d y,
$$

$p(t)$ defined as in Lemma 5.4, it follows that

$$
\left(\rho\left(r_{m+1, x}\right)(\lambda)\right)^{1 / 2} \leqq c(M) I_{m}(x ; \lambda)
$$

for $\lambda^{2} \leqq 1+M$.

For $h \in L_{\infty}\left(R^{n}\right)$ consider the operator

$$
(J h)(x)=\int_{R^{n}}|V(y)| p(|x-y|) h(y) d y .
$$

With the aid of Sobolev's inequality (cf. [1, Part II, §5.3c]) it can readily be seen that

$$
(J h)(x) \leqq C_{q}|V||h|_{\infty},
$$

$C_{q}$ depending only on $q$ and $n$. Thus, we may absorb it into $\Lambda$ to obtain from (*)

$$
I_{m}(x ; \lambda) \leqq c(M)\left[J I_{m-1}(\cdot ; \lambda)\right](x) \leqq c(M)|V|\left|I_{m-1}(\cdot ; \lambda)\right|_{\infty} .
$$

Since $r_{0, x}=P \Psi^{s}{ }_{x}$, we obtain, from $\left(^{* *}\right)$ and the $a=0$ case of formula (5.3), that $I_{0}(x ; \lambda) \leqq C_{q} M^{n-3}|V|$. Thus $I_{m}(x ; \lambda) \leqq[c(M)|V|]^{m+1}, \lambda^{2}<M^{2}+1$, giving, by $\left(^{*}\right)$,

$$
\left(\rho\left(r_{m, x}\right)(\lambda)\right)^{1 / 2} \leqq c(M)^{m+1}|V|^{m}, \quad \lambda^{2}<M^{2}+1 .
$$

The theorem now follows by summing over all $m$ for the first formula in the statement, over all $m \geqq 1$ for the second formula. Recall the definition of $\|\cdot\| \|_{(N)}$ and Lemma 2.3(iii).

LeMmA 6.2. Let $V^{\prime}$ also satisfy $\left|V^{\prime}\right|<c(M)^{-1}$. Then, for $\lambda^{2}<M^{2}+1$,

$$
\int_{R^{n}}|V(x)|\left(\rho\left(Y^{*} \Psi_{x}^{\varepsilon}\right)(\lambda)\right)^{1 / 2} d x \leqq \frac{c(M)^{2}|V| \cdot\left|V^{\prime}-V\right|_{1}}{[1-c(M)|V|]\left[1-c(M)\left|V^{\prime}\right|\right]}
$$

where $Y=\Omega^{8}\left(V^{\prime}\right)-\Omega^{8}(V)$.

Proof. For a real-valued function $h$ in $L_{1} \cap L_{q}$, let $h_{1}, h_{2}$ be such that

$$
h_{1}(x) h_{2}(x)=h(x), \quad h_{1}(x)^{2}=h_{2}(x)^{2}=h(x) .
$$

Recall that setting $f_{x}^{\varepsilon}(h)=h_{1}(x) \Phi_{x}^{\varepsilon}, g^{\varepsilon}{ }_{x}(h)=h_{2}(x) \Psi^{\varepsilon}{ }_{x}$, we obtain

$$
A_{\varepsilon}(h)=\int_{R^{n}}\left(, g_{x}^{\varepsilon}(h)\right) f_{x}^{\varepsilon}(h) d x
$$


Set

$$
\begin{aligned}
& f^{\varepsilon}{ }_{x}(V)=f^{\varepsilon}{ }_{x}, \quad f^{\varepsilon}{ }_{x}\left(V^{\prime}\right)=f^{\varepsilon}{ }_{x}^{\prime}, \quad f^{8}{ }_{x}\left(V^{\prime}-V\right)=n_{x} ; \\
& g_{x}^{\varepsilon}(V)=g_{x}^{\varepsilon}, \quad g_{x}^{\varepsilon}\left(V^{\prime}\right)=g_{x}^{\varepsilon}, \quad g_{x}^{\varepsilon}\left(V^{\prime}-V\right)=m_{x} \text {. }
\end{aligned}
$$

Notice that, by the equation of Theorem 5.5,

$$
\begin{aligned}
Y^{*} & =\left[\Omega^{\varepsilon}\left(V^{\prime}\right)-\Omega^{\varepsilon}(V)\right]^{*} \\
& =\left\{\Gamma\left[A_{\varepsilon}\left(V^{\prime}\right) \Omega^{\varepsilon}\left(V^{\prime}\right)-A_{\varepsilon}(V) \Omega^{\varepsilon}(V)\right]\right\}^{*} \\
& =-\Gamma\left\{\left[A_{\varepsilon}\left(V^{\prime}-V\right) \Omega^{\varepsilon}\left(V^{\prime}\right)\right]^{*}\right\}-\Gamma\left\{\left[A_{\varepsilon}(V) Y\right]^{*}\right\} .
\end{aligned}
$$

Using $(\rho(u+v))^{1 / 2} \leqq(\rho(u))^{1 / 2}+(\rho(v))^{1 / 2}$, we obtain, proceeding as in the case of formula (2.8),

$$
\left(\rho\left(Y^{*} \Psi_{x}^{s}\right)(\lambda)\right)^{1 / 2} \leqq I_{1}(x ; \lambda)+I_{2}(x ; \lambda),
$$

with

$$
\begin{aligned}
I_{1}(x ; \cdot) & =\int_{R^{n}}\left|\sigma\left(\Psi_{x}^{\varepsilon}, n_{y}\right)\right|\left(\rho\left(\Omega^{\varepsilon}\left(V^{\prime}\right)^{*} m_{y}\right)\right)^{1 / 2} d y \\
& =\int_{R^{n}}\left|V^{\prime}(y)-V(y)\right||\sigma(x, y ; \cdot)|\left(\rho\left(\Omega^{\varepsilon}\left(V^{\prime}\right) \Psi^{\varepsilon} y\right)\right)^{1 / 2} d y, \\
I_{2}(x ; \cdot) & =\int_{R^{n}}\left|\sigma\left(\Psi^{\varepsilon}{ }_{x}, f_{y}^{\varepsilon}\right)\right|\left(\rho\left(Y^{*} g_{y}^{\varepsilon}\right)\right)^{1 / 2} d y \\
& =\int_{R^{n}}|V(y)||\sigma(x, y ; \cdot)|\left(\rho\left(Y^{*} \Psi_{y}^{s}\right)\right)^{1 / 2} d y .
\end{aligned}
$$

It follows now from Lemmas 5.4 and 6.1 that

$$
I_{1}(x ; \lambda) \leqq \frac{c(M)^{2}}{1-c(M)\left|V^{\prime}\right|}\left|\left(V^{\prime}-V\right)(y)\right| \cdot p(|x-y|) d y .
$$

Clearly, $p(|x|)=g_{1}(x)+g_{2}(x)$, with $g_{1} \in L_{q /(q-1)}, g_{2} \in L_{\infty}$. Hence

$$
\begin{aligned}
\int_{R^{n}} \int_{R^{n}}\left|V^{\prime}(y)-V(y)\right||V(x)| & p(|x-y|) d x d y \\
& \leqq\left|V^{\prime}-V\right|_{1}\left(|| V\left|* g_{1}\right|_{\infty}+|| V\left|* g_{2}\right|_{\infty}\right) \\
& \leqq C_{q}\left|V^{\prime}-V\right|_{1}|V| .
\end{aligned}
$$

Multiplying both sides of the inequality in (*') by $|V(x)|$ and integrating with respect to $x$, we get by $\left({ }^{* \prime \prime}\right)$, for $\lambda^{2}<M^{2}+1$,

$$
\int_{R^{n}} I_{1}(x ; \lambda)|V(x)| d x \leqq \frac{c(M)^{2}}{1-c(M)\left|V^{\prime}\right|}|V|\left|V^{\prime}-V\right|_{1} .
$$

By Lemma 5.4, for $\lambda^{2}<M^{2}+1$,

$$
I_{2}(x ; \cdot) \leqq c(M) \int_{R^{n}}|V(y)| p(|x-y|)\left(\rho\left(Y^{*} \Psi_{y}^{8}\right)\right)^{1 / 2} d y .
$$


Since $\int_{R^{n}}|V(x)| p(|x-y|) d x \leqq|| V\left|* g_{1}\right|_{\infty}+|| V\left|* g_{2}\right|_{\infty} \leqq C_{q}|V|$, we obtain from $(* *)$

$\left({ }^{* * \prime}\right) \quad \int_{R^{n}}|V(x)| I_{2}(x ; \lambda) d x \leqq c(M)|V| \int_{R^{n}}|V(y)|\left(\rho\left(Y^{*} \Psi_{y}^{8}\right)(\lambda)\right)^{1 / 2} d y$.

By $(*),(* m)$ and $(* *)$

$$
\begin{aligned}
& \int_{R^{n}}|V(x)|\left(\rho\left(Y^{*} \Psi_{x}^{\varepsilon}\right)(\lambda)\right)^{1 / 2} d x \\
& \quad \leqq \frac{c(M)|V|}{1-c(M)\left|V^{\prime}\right|}\left|V^{\prime}-V\right|_{1}+|V| c(M) \int_{R^{n}}|V(y)|\left(\rho\left(Y^{*} \Psi_{y}^{\varepsilon}\right)(\lambda)\right)^{1 / 2} d y
\end{aligned}
$$

and the lemma follows.

7. Fix $a \geqq(n-1) / 2$ and denote by $\boldsymbol{B}=B\left(\boldsymbol{H}, \boldsymbol{H}_{-a}\right)$ the Banach space of all bounded linear operators having domain $\boldsymbol{H}$, range included in $\boldsymbol{H}_{-a}$, with the usual operator norm. Since $\boldsymbol{H}$ is a dense subspace of the Hilbert space $\boldsymbol{H}_{-a}$, and since the norm in $\boldsymbol{H}$ dominates the norm in $\boldsymbol{H}_{-a}$, every bounded operator on $\boldsymbol{H}$ may be interpreted as an element of $\boldsymbol{B}$.

Let $T$ be any operator, domain of $T=\boldsymbol{H}$. We reserve the symbol $|T|$ to denote its norm as an element of $\boldsymbol{B}$, i.e.

$$
\begin{aligned}
|T| & =\sup \left\{\|T u\|_{-a}: u \in \boldsymbol{H},\|u\|=1\right\} \\
& =\sup \left\{|(T u, v)|: u \in \boldsymbol{H}, v \in \boldsymbol{H}_{a},\|u\|=\|v\|_{a}=1\right\} .
\end{aligned}
$$

The second equality is a consequence of the duality between $\boldsymbol{H}_{\boldsymbol{a}}$ and $\boldsymbol{H}_{-a}$ determined by the inner product of $\boldsymbol{H}$. A typical example (for our purposes) of element of $\boldsymbol{B}$ is given by the following

LEMma 7.1. Let $V \in L_{1}\left(R^{n}\right)$, let $T$ be a bounded operator on $\boldsymbol{H}$ such that $\left\|T^{*} \Psi^{\varepsilon}{ }_{x}\right\|$ is bounded by a constant $K$. Define an operator $Q_{\varepsilon}$ by setting

$$
\left(Q_{\varepsilon} u, v\right)=\int_{-\infty}^{+\infty}\left(\exp (i t H) A_{\varepsilon} T \exp (-i t H) u, v\right) d t
$$

for $u \in H, v \in H_{a} ; A_{\varepsilon}=A_{\varepsilon}(V)$. Then $\left|Q_{\varepsilon}\right| \leqq K \Lambda|V|_{1}$.

Proof. Notice that $\left\|\Phi^{\varepsilon}\right\|_{-a} \leqq \Lambda$ by (5.2). Expressing $A_{\varepsilon}(V)$ by an integral, as usual, and using part c of Lemma 2.2 , it is easily seen that

$$
\left|\left(Q_{\varepsilon} u, v\right)\right| \leqq \int_{R^{n}} \int_{-\infty}^{+\infty}|V(x)|\left|\rho\left(u, T^{*} \Psi_{x}^{\varepsilon}\right)(\lambda)\right|\left|\rho\left(\Phi^{\varepsilon}, v\right)(\lambda)\right| d x d \lambda
$$

The lemma follows now from (5.1), Hölder's inequality and the hypothesis.

For $V \in L_{1}$, define an operator $T_{0}{ }^{8}(V)$ by

$$
T_{0}^{\varepsilon}(V)=-i \int_{-\infty}^{+\infty} \exp (i t H) A_{\delta}(V) \exp (-i t H) d t .
$$

By Lemma 7.1, $T_{0}{ }^{8}(V) P \in B,\left|T_{0}{ }^{8}(V) P\right| \leqq \Lambda|V|_{1}$. The operator $S_{\varepsilon}(V) P$ is also in $B$ 
(set $T=\Omega^{\varepsilon}$ - in Lemma 7.1, recall Lemma 5.6) and so are the operators $S(V) P$, $T_{0}(V) P$ defined by

$$
\begin{gathered}
S(V) P=\text { w-lim }(\varepsilon \downarrow 0) S_{\varepsilon}(V) P, \\
T_{0}(V) P=\text { w-lim }(\varepsilon \downarrow 0) T_{0}{ }^{8}(V) P .
\end{gathered}
$$

That the limit in (7.1) exists is a consequence of Lemma 5.6. The existence of the limit in (7.2) is proved similarly. It is clear that this new definition of $S(V) P$ coincides with the one given in (4.3). This is also a consequence of Lemma 5.6.

THEOREM 7.2. Let $V, V^{\prime} \in L_{1} \cap L_{q}, q>n / 2,|V|<c(M)^{-1},\left|V^{\prime}\right|<c(M)^{-1}$. Then

$$
\begin{aligned}
\mid P S\left(V^{\prime}\right) P-P S(V) P-P T_{0} & \left(V^{\prime}-V\right) P \mid \\
& \leqq \frac{c(M)^{2}}{1-c(M)\left|V^{\prime}\right|}\left[\left|V^{\prime}\right|+\frac{|V|}{1-c(M)|V|}\right]\left|V^{\prime}-V\right|_{1} .
\end{aligned}
$$

Proof. Set $X=\Omega^{\varepsilon}{ }_{-}\left(V^{\prime}\right)-P$ and define $Y$ as in Lemma 6.2; we use the same notation as in the proof of Lemma 6.2. Set

Then

$$
D_{\varepsilon}=P\left[S_{\varepsilon}\left(V^{\prime}\right)-S_{\varepsilon}(V)-T_{0}{ }^{8}\left(V^{\prime}-V\right)\right] P \text {. }
$$

$$
\begin{aligned}
D_{\varepsilon}=-i\left(\int_{-\infty}^{+\infty} \exp (i t H) A_{\varepsilon}\left(V^{\prime}-V\right) X\right. & \exp (-i t H) d t \\
& \left.\quad+\int_{-\infty}^{+\infty} \exp (i t H) A_{\varepsilon}(V) Y \exp (-i t H) d t\right) .
\end{aligned}
$$

To estimate $\left(D_{\varepsilon} u, v\right)$ we may assume $P u=u, P v=v$.

$$
\begin{aligned}
i\left(D_{\varepsilon} u, v\right)= & \int_{-\infty}^{+\infty}\left(A_{\varepsilon}\left(V^{\prime}-V\right) X \exp (-i t H) u, \exp (-i t H) v\right) d t \\
& +\int_{-\infty}^{+\infty}\left(A_{\varepsilon}(V) Y \exp (-i t H) u, \exp (-i t H) v\right) d t \\
= & \int_{R^{n}} \int_{-\infty}^{+\infty}\left(\exp (-i t H) u, X^{*} m_{x}\right)\left(n_{x}, \exp (-i t H) v\right) d x d t \\
& +\int_{R^{n}} \int_{-\infty}^{+\infty}\left(\exp (-i t H) u, Y^{*} g^{\varepsilon}{ }_{x}\right)\left(f^{\varepsilon}{ }_{x}, \exp (-i t H) v\right) d x d t \\
= & \int_{R^{n}} \int_{-\infty}^{+\infty}\left[V^{\prime}(x)-V(x)\right]\left(e^{-i t H} u, X^{*} \Psi^{\varepsilon}{ }_{x}\right)\left(\Phi^{\varepsilon}{ }_{x}, e^{-i t H} v\right) d x d t \\
& +\int_{R^{n}} \int_{-\infty}^{+\infty} V(x)\left(e^{-i t H} u, Y^{*} \Psi^{\varepsilon}{ }_{x}\right)\left(\Phi^{\varepsilon}{ }_{x}, e^{-i t H} v\right) d x d t
\end{aligned}
$$

Thus, by Lemma $2.2 \mathrm{c}$ and inequality (5.1), for $N^{2}=M^{2}+1$,

$$
\begin{aligned}
&\left(D_{\varepsilon} u, v\right) \leqq \int_{R^{n}}\left|V^{\prime}(x)-V(x)\right| \cdot\left|\left\|X ^ { * } \Psi _ { x } ^ { \varepsilon } \left|\left\|_{(N)} \cdot\left|\left\|\Phi^{\varepsilon}{ }_{x}\right\|\right|_{-a} \cdot\right\| u\|\cdot\| v \|_{\alpha} d x\right.\right.\right. \\
&+\int_{-\infty}^{+\infty}(\rho(u)(\lambda))^{1 / 2}\left(\rho^{a}(v)(\lambda)\right)^{1 / 2} \int_{R^{n}}|V(x)|\left(\rho\left(Y^{*} \Psi_{x}^{\varepsilon}\right)\right)^{1 / 2}(\lambda) \\
& \cdot\left(\rho^{-a}\left(\Phi^{\varepsilon}{ }_{x}\right)(\lambda)\right)^{1 / 2} d x d \lambda
\end{aligned}
$$


We estimate the first term of the last member of this inequality by Lemma 6.1, the other term by Lemma 6.2 . Since $\left\|\mid \Phi_{x}^{\varepsilon}\right\|_{-a}$ is bounded by $\Lambda$, the theorem follows.

LEMMA 7.3. Let $0<\eta$. Set $f_{\eta}(r)=0$ for $r>\eta,=r^{(2-n) / 2}\left(1+r^{2}\right)^{-1 / 4}$ for $0<r<\eta$. Let $u(t)=u(\cdot, t)=\exp (i t B) \psi_{\eta}$, with $\hat{\psi}_{\eta}(\xi)=f_{\eta}(|\xi|)$.

Then

$$
\int_{-\infty}^{+\infty}|u(x, t)|^{2} d t \geqq \Lambda \eta^{2}\left(\frac{J_{k}(\eta|x|)}{|x|^{k}}\right)^{2}
$$

with $J_{k}$ the kth Bessel function of the first kind, $k=(n-2) / 2$.

Proof.

$$
\begin{aligned}
u(x, t) & =\int_{R^{n}} e^{i\langle x, \xi\rangle} \exp \left(i t\left(1+|\xi|^{2}\right)^{1 / 2}\right) \hat{\psi}(\xi) d \xi \\
& =|x|^{-k} \int_{0}^{\infty} r^{n / 2} \exp \left(i t\left(1+r^{2}\right)^{1 / 2}\right) f(r) J_{k}(r|x|) d r
\end{aligned}
$$

(We drop $\eta$ as a subindex during this proof.)

Multiplying the last expression by its complex conjugate, integrating with respect to $t$ from $-R$ to $+R$, we get, after a change of variables,

$$
|x|^{2-n} \int_{-\infty}^{+\infty}|u(x, t)|^{2} d t=\int_{2}^{\infty} d v \int_{-v+2}^{v-2} d \theta \frac{\sin R \theta}{\theta} g(\alpha, \beta)\left(v^{2}-\theta^{2}\right),
$$

with

$$
\begin{aligned}
g(\alpha, \beta) & =(\alpha \beta)^{n-1} f(\alpha) f(\beta) J_{k}(\alpha|x|) J_{k}(\beta|x|), \\
\alpha & =\left(\frac{1}{2}\right)\left((\theta+v)^{2}-4\right)^{1 / 2}, \quad \beta=\left(\frac{1}{2}\right)\left((\theta-v)^{2}-4\right)^{1 / 2} .
\end{aligned}
$$

Since the inner integral is a Dirichlet integral, letting $R \rightarrow+\infty$, by the Lebesgue dominated convergence theorem (which is used to interchange the limiting process with the outer integral), we obtain, after a change of variables,

$$
\begin{aligned}
|x|^{n-2} \int_{-\infty}^{+\infty}|u(x, t)|^{2} d t & =8 \int_{0}^{\infty} r^{n-1}\left[1+r^{2}\right]^{1 / 2}[f(r)]^{2}\left[J_{k}(r|x|)\right]^{2} d r \\
& =8 \int_{0}^{n} r\left[J_{k}(r|x|)\right]^{2} d r .
\end{aligned}
$$

The last integral may be evaluated explicitly (cf. [4, (10), p. 90]). We obtain

$$
|x|^{n-2} \int_{-\infty}^{+\infty}|u(x, t)|^{2} d t=4 \eta^{2}\left[J_{k}(\eta|x|)-J_{k+1}(\eta|x|) J_{k-1}(\eta|x|)\right],
$$

and the result becomes now exactly the statement of Szasz' inequality, as stated on p. 12 of [4].

Consider the function $F(r)=\left(J_{k}(r) / r^{k}\right)^{2}, k=(n-2) / 2$. This function takes on the value $\omega_{n}^{2}\left(\omega_{n}=\right.$ area of the unit sphere in $\left.R^{n}\right)$ at $r=0$, and is strictly decreasing in 
the interval $\left[0, \gamma_{k}\right]$, with $\gamma_{k}>(k(k+2))^{1 / 2}=\left(\frac{1}{2}\right)\left(n^{2}-4\right)^{1 / 2}$. This is an easy consequence of the recurrence relations for Bessel functions of the first kind [4, (51), p. 11] and the properties of $\gamma_{v}$, the first positive zero of $J_{v}[4, \mathrm{pp} .59-60]$.

Now choose $0<M, R, z_{0}$ such that $z_{0}<\min \left(M R, \gamma_{k}\right)$. Set $c\left(R, z_{0}\right)$ $=\left(\Lambda R^{a+1} /\left(R^{2}+z_{0}^{2}\right)^{(a+1) / 2}\right) R^{2-n}\left(J_{k}\left(z_{0}\right)\right)^{2}$ and $\eta=z_{0} / R<M$.

Then, if $\psi=\psi_{\eta}$ is as in Lemma 7.3, it is clear that $u=\{\psi, i B \psi\}, v=\left\{i B^{-1} \psi, \psi\right\}$ are in all spaces $\boldsymbol{H}_{b}\left(b \in R^{1}\right)$, with $\|u\| \leqq \Lambda \eta\left(1+\eta^{2}\right)^{1 / 4},\|v\|_{a} \leqq \Lambda \eta\left(1+\eta^{2}\right)^{(2 a+1) / 4}$. Since $\eta<M, P u=u$ and $P v=v$. Also

$$
\left(P T_{0}(V) P u, v\right)=\left(T_{0}(V) u, v\right)=\int_{R^{n}} V(x) \int_{-\infty}^{+\infty}|u(x, t)|^{2} d x d t,
$$

with $u(x, t)$ as in Lemma 7.3. Thus, by Lemma 7.3, assuming $V(x) \geqq 0$ and $F(r)$ as above

$$
\begin{aligned}
\Lambda^{2} \eta^{2}\left(1+\eta^{2}\right)^{(a+1) / 2}\left|P T_{0}(V) P\right| & \geqq\left(P T_{0}(V) P u, v\right) \\
& \geqq \Lambda \eta^{2} \int_{R^{n}} V(x) F(\eta|x|) d x \cdot \eta^{2 k} \\
& \geqq \Lambda \eta^{2} \int_{|x|<R} V(x) F(\eta|x|) d x \cdot \eta^{2 k} \\
& \geqq F\left(z_{0}\right) \eta^{n-2} \int_{|x|<R} V(x) d x .
\end{aligned}
$$

Carrying out the cancellations, we get

$$
\left|P T_{0}(V) P\right| \geqq c\left(R, z_{0}\right) \int_{|x|<R} V(x) d x .
$$

Since all operators involved are translation invariant, we have actually proved

THEOREM 7.4. Let $V \geqq 0 ; M, R, z_{0}, c\left(R, z_{0}\right)$ as above; $\Omega_{R}$ a compact set of diameter $\leqq R$. Then

$$
\left|P T_{0}(V) P\right| \geqq c\left(R, z_{0}\right) \int_{\Omega_{R}} V(x) d x .
$$

Let $V, V^{\prime} \in L_{1} \cap L_{q}(q>n / 2)$. Assume $|V|<c(M)^{-1},\left|V^{\prime}\right|<c(M)^{-1}$. If $V^{\prime}-V \geqq 0$, or $V^{\prime}-V \leqq 0$, and $S\left(V^{\prime}\right)=S(V)$, then combining Theorems 7.2 and 7.4 we get

$$
\frac{c(M)}{1-c(M)\left|V^{\prime}\right|}\left[\left|V^{\prime}\right|+\frac{|V|}{1-c(M)|V|}\right]\left|V^{\prime}-V\right|_{1} \geqq c\left(R, z_{0}\right) \int_{\Omega_{R}}\left|V^{\prime}(x)-V(x)\right| d x .
$$

From this, our main result is immediate.

THEOREM 7.5. Let $V, V^{\prime} \in L_{1} \cap L_{q}(q>n / 2)$. For each $R>0$ there exists $a$ constant $d=d(R, n, q)$ such that if $|V|<d,\left|V^{\prime}\right|<d$, and $V^{\prime}-V$ is either $\geqq 0$ or $\leqq 0$ and of compact support $\Omega_{R}$, with $\operatorname{diam}\left(\Omega_{R}\right) \leqq R$, then $S(V)=S\left(V^{\prime}\right)$ implies $V=V^{\prime}$. In particular, if $V \geqq 0$ or $V \leqq 0$, diam (supp $V) \leqq R$, then $S(V)=I$ implies either $V=0$, or $|V| \geqq d$. 
To conclude, let us notice that $c(M)>1$, but can be brought as near to 1 as desired. But since we want $c\left(z_{0}, R\right)$ as large as possible and since $c(0, R)=0$, it is convenient to have a not too small $z_{0}$. Since $0<z_{0}<M R, M$ small forces $R$ to be large, and vice versa. It is also clear that the operator $S(V)$ is the usual scattering operator, in the sense that it relates incoming to outgoing solutions. This, at least for solutions with initial data having Fourier transform of compact support, follows easily from Lemma 5.6.

\section{REFERENCES}

1. L. Bers, F. John and M. Schechter, Partial differential equations, Lectures in Appl. Math., vol. 3, Interscience, New York, 1964. MR 29 \#346.

2. J. M. Chadam, The asymptotic behavior of the Klein-Gordon equation with external potential, J. Math. Anal. Appl. 31 (1970), 334-348. MR 41 \#7487.

3. A. Degasperis, On the inverse problem for the Klein-Gordon s-wave equation, J. Mathematical Phys. 11 (1970), 551-567. MR 41 \#1324.

4. A. Erdélyi, W. Magnus, F. Oberhettinger and F. G. Tricomi, Higher transcendental functions. Vol. 2, McGraw-Hill, New York, 1953. MR 15, 419.

5. T. Kato, Perturbation theory for linear operators, Die Grundlehren der math. Wissenschaften, Band 132, Springer-Verlag, New York, 1966. MR 34 \#3324.

6. P. D. Lax and R. S. Phillips, Scattering theory, Pure and Appl. Math., vol. 26, Academic Press, New York, 1967. MR 36 \#530.

7. S. Nelson, On some solutions to the Klein-Gordon equation related to an integral of Sonine, Trans. Amer. Math. Soc. 154 (1971), 227-237.

8. R. Newton, Scattering theory of waves and particles, McGraw-Hill, New York, 1966. MR 36 \#4875.

9. E. T. Whittaker and G. N. Watson, $A$ course of modern analysis, 4th ed., Cambridge Univ. Press, New York, 1962. MR 31 \#2375.

Department of Mathematics, Florida Atlantic University, Boca Raton, Florida 33432 